\title{
Características Morfológicas y Maduración en Mujeres Kayakistas Jóvenes de Aguas Tranquilas y Slalom
}

\author{
Morphological Characteristics and Maturity Status of Young Female Sprint and Slalom Kayakers
}

\author{
"Fernando Alacid; ${ }^{* *}$ José María Muyor; "Raquel Vaquero \& ${ }^{* * * *}$ Pedro Ángel López-Miñarro
}

ALACID, F.; MUYOR, J. M.; VAQUERO, R. \& LÓPEZ-MIÑARRO, P. A. Características morfológicas y maduración en mujeres kayakistas jóvenes de aguas tranquilas y slalom. Int. J. Morphol., 30(3):895-901, 2012.

RESUMEN: El objetivo de este trabajo fue describir y comparar las características antropométricas, maduración, somatotipo y proporcionalidad de mujeres kayakistas de aguas tranquilas y slalom. Cincuenta palistas de 13 a 14 años de edad (26 de aguas tranquilas y 24 de slalom), fueron valoradas usando una batería de 29 medidas antropométricas. Se calculó el porcentaje de grasa, el somatotipo, los valores $\mathrm{Z}$ utilizando la estrategia Phantom de proporcionalidad y la maduración expresada como los años hasta/desde la edad de máxima velocidad de crecimiento en altura. Las palistas de aguas tranquilas se caracterizaron por una mayor talla y talla sentado que las de slalom, lo que podría interpretarse como un mayor desarrollo madurativo de las primeras. Los valores de proporcionalidad fueron similares en la mayoría de las variables analizadas. Ambos grupos de kayakistas presentaron un somatotipo medio clasificado como mesomorfo endomorfo, siendo las palistas de slalom las que presentaron un somatotipo más heterogéneo. La información aportada en este estudio puede ser utilizada como guía en el proceso de detección de talentos en mujeres kayakistas de aguas tranquilas y slalom.

PALABRAS CLAVE: Antropometría; Somatotipo; Proporcionalidad; Piragüismo/canotaje.

\section{INTRODUCCIÓN}

Un amplio número de investigaciones han sido utilizadas para diferenciar las características antropométricas de deportistas en función de la posición de juego en deportes colectivos como el fútbol (Hencken \& White, 2006; Sutton et al., 2009), voleibol (Duncan et al., 2006; Toledo et al., 2010), baloncesto (Ackland et al., 1997; Carter et al., 2005; Goncetas \& Landor, 2005), etc, encontrando diferencias significativas entre las diferentes posiciones de juego. Asimismo, diversas investigaciones han identificado diferentes perfiles antropométricos en deportes individuales en función de la disciplina atlética (Vucetic et al., 2008), estilos de natación (Carter \& Ackland, 1994) así como de las categorías de peso ligero y pesado en remo (Jürimäe \& Jürimäe, 2002; Kerr et al., 2007).

En piragüismo o canotaje, las comparaciones de tipo antropométrico se han centrado en la búsqueda de diferencias entre las disciplinas de canoa y kayak de aguas tranqui- las (Hirata, 1977; Misigoj-Durakovic \& Heimer, 1992) y de slalom (Ridge et al., 2007). Más recientemente, diversos trabajos han comparado las características antropométricas e índices corporales de palistas jóvenes con un año de diferencia de edad (Alacid, et al., 2011a, b), así como la proporcionalidad con respecto a palistas de elite (Alacid et al., 2011b).

Sin embargo, son escasos los trabajos que comparan las características morfológicas de las mujeres kayakistas de las especialidades de aguas tranquilas y slalom. Únicamente Ridge et al., en la discusión de su investigación, realiza comparaciones entre datos obtenidos en palistas de elite de slalom y los datos de referencia en palistas de aguas tranquilas del mismo nivel de competencia (Ackland et al., 2003), encontrando similitudes en cuanto a la talla y un menor peso y adiposidad en las palistas de slalom.

\footnotetext{
Facultad de Ciencias de la Actividad Física y el Deporte, Universidad Católica San Antonio, España.

** Área de Expresión Corporal. Facultad de Ciencias de la Educación, Universidad de Almería, España.

**** Departamento de Expresión Plástica, Musical y Dinámica, Facultad de Educación, Universidad de Murcia, España.

Este estudio ha sido financiado con una ayuda de la Fundación Séneca-Agencia de Ciencia y Tecnología de la Región de Murcia (II PCTRM 20072010) con $n^{\circ}$ 11951/PI/09 (Evolución de las curvaturas sagitales del raquis, extensibilidad isquiosural, dolor lumbar y características antropométricas de piragüistas de élite).
} 
A pesar de que, en ambos casos, los kayakistas palean de forma alternativa por ambos lados en una posición de sentado, utilizando una pala de doble hoja, las diferencias entre las especialidades de aguas tranquilas y slalom son notables. En aguas tranquilas, el palista compite a lo largo de una calle acotada por boyas en una lámina de agua sin obstáculos, con el objetivo de recorrer una determina distancia en el menor tiempo posible; mientras que en slalom, debe completar un recorrido, en el menor tiempo posible, de aguas bravas lleno de dificultades, debiendo pasar por determinadas puertas colocadas a favor y en contra de la corriente, evitando tocarlas o no pasarlas, puesto que este tipo de infracciones conllevaría una importante penalización en tiempo (RFEP, 2010).

Dadas las diferencias entre ambas especialidades y la escasez de trabajos que aborden las posibles diferencias morfológicas entre palistas jóvenes de aguas tranquilas y slalom, el objetivo del presente trabajo fue describir y comparar las características antropométricas, maduración, somatotipo y proporcionalidad de mujeres kayakistas de aguas tranquilas y slalom, con el fin de establecer un perfil antropométrico de referencia que pueda ser usado en el proceso de detección de talentos deportivos en dicha disciplina.

\section{MATERIAL Y MÉTODO}

Un total de 50 mujeres kayakistas de categoría infantil (edad: 13 - 14 años) participaron en este estudio. Veintiséis competían en la especialidad de aguas tranquilas y 24 en la de slalom sobre aguas bravas. Todas ellas fueron seleccionadas por encontrarse entre los mejores de su categoría, para su asistencia a las Concentraciones Nacionales de Infantiles en los años 2008 y 2009, realizadas dentro del Programa Nacional de Tecnificación de Infantiles del Consejo Superior de Deportes y la Real Federación Española de Piragüismo.

Los padres y los deportistas fueron informados de los objetivos y métodos del estudio y se obtuvo un consentimiento informado de sus tutores. El estudio fue aprobado por el Comité Ético y de Investigación de la Universidad de Murcia.

Las variables (descritas en la tabla I) fueron tomadas por un antropometrista acreditado de nivel II siguiendo las indicaciones descritas por la ISAK (International Society for the Advancement of Kinanthropometry) (Marfell-Jones et al., 2006). Las medidas se tomaron dos o tres veces, si la diferencia entre las dos primeras era su- perior al $5 \%$ en pliegues y al $1 \%$ en el resto de medidas, tomando la media o la mediana, respectivamente, para realizar los análisis posteriores.

Para la determinación del peso se utilizó una báscula SECA 862 (SECA, Alemania) de 100 g de precisión; para los pliegues un plicómetro Harpenden (British Indicators, UK) de 0,2 $\mathrm{mm}$ de precisión; para la envergadura y los perímetros una cinta métrica inextensible milimetrada Lufkin W606PM (Lufkin, EE.UU.); y para la talla, talla sentado y diámetros, un antropómetro SiberHegner GPM (Suiza), ambos instrumentos con una precisión de $0,1 \mathrm{~cm}$.

Las ecuaciones de Carter \& Heath (1990) se utilizaron para calcular cada uno de los componentes del somatotipo y Estrategia del Phantom (Ross \& MarfellJones, 1991) para la determinación de los valores $\mathrm{Z}$ de cada una de las variables. También se calculó el índice de masa corporal y los sumatorios de seis y ocho pliegues cutáneos. Los perímetros corregidos se obtuvieron mediante la siguiente fórmula: perímetro corregido $=$ perímetro ( $\pi *$ pliegue cutáneo). Se utilizaron las ecuaciones de Slaughter et al. (1988) para la determinación del porcentaje de grasa corporal. Se eligieron estas ecuaciones al considerarse las más precisas para la determinación del porcentaje graso en adolescentes (Rodriguez et al., 2005). La masa libre de grasa se obtuvo tras sustraer la masa grasa a la masa total.

La estimación de la maduración se realizó siguiendo el procedimiento descrito por Mirwald et al. (2002) para determinar el tiempo, expresado en años, que ha pasado (valores positivos) o que falta (valores negativos) para llegar a la edad de máxima velocidad de crecimiento en altura (tomando este acontecimiento como un indicador del proceso de maduración corporal durante la adolescencia).

Los resultados fueron analizados de forma independiente para las mujeres kayakistas de aguas tranquilas y slalom. Se realizó un análisis descriptivo de cada una de las variables, expresando sus valores en media \pm desviación típica. Se comprobó la normalidad de los datos utilizando el test de Shapiro-Wilk, y dependiendo de los resultados, se realizaron análisis paramétricos o no paramétricos. Para las variables que mostraron una distribución normal se utilizó una prueba t para muestras independientes, mientras que para aquéllas cuya distribución no seguía una distribución normal se aplicó la prueba U de Mann-Whitney. Para todos los test estadísticos el nivel de significación se situó en un valor de $\mathrm{p}<0,05$. Todas las pruebas estadísticas fueron realizadas usando el paquete estadístico SPSS para Windows (versión 15,0). 


\section{RESULTADOS Y DISCUSIÓN}

La Tabla I muestra las características antropométricas y las diferencias entre las mujeres kayakistas infantiles de las especialidades de aguas tranquilas y slalom. Las kayakistas de aguas tranquilas fueron significativamente superiores en talla y talla sentado a las palistas de slalom, por lo que la comparación de los valores obtenidos con las tablas de crecimiento de la población española (Carrascosa et al., 2008), sitúan a las palistas de aguas tranquilas en el percentil 80 y en el 50 a las de slalom. Estas diferencias, pueden estar motivadas por el significativamente mayor nivel de maduración en las palistas de aguas tranquilas (Tabla III) y por el hecho de que los criterios de selección para asistir a las Concentraciones Nacionales se basan, únicamente, en el rendimiento en el agua. Por todo ello, y dadas las características de cada una de las especialidades, la importancia del desarrollo físico podría tener un papel determinante en el rendimiento en palistas de aguas tranquilas de esta categoría, mientras que el nivel técnico podría ser el aspecto más importante en la especialidad de slalom.

En cuanto al peso corporal, no se encontraron diferencias significativas, situando los valores de ambas especialidades en torno al percentil 50 y 75 (Carrascosa et al.), si bien las palistas de slalom obtuvieron un peso medio in-

Tabla I. Comparación y descripción de las características antropométricas de las mujeres kayakistas jóvenes de aguas tranquilas y slalom.

\begin{tabular}{|c|c|c|c|}
\hline Variable & $\begin{array}{l}\text { Aguas Tranquilas } \\
\quad(\mathrm{n}=\mathbf{2 6})\end{array}$ & Slalom $(n=24)$ & Valor $-p$ \\
\hline Edad (años) & $13,70 \pm 0,61$ & $13,78 \pm 0,54$ & 0,606 \\
\hline Peso (kg) & $56,58 \pm 8,66$ & $52,98 \pm 7,49$ & 0,123 \\
\hline Sumatorio de 6 pliegues (mm) & $96,87 \pm 23,81$ & $90,51 \pm 26,18$ & 0,373 \\
\hline Sumatorio de 8 pliegues (mm) & $121,88 \pm 30,88$ & $112,70 \pm 32,53$ & 0,311 \\
\hline Talla $(\mathrm{cm})$ & $163,92 \pm 5,52$ & $158,03 \pm 4,22$ & $<0,001$ \\
\hline Talla sentado $(\mathrm{cm})$ & $86,61 \pm 2,73$ & $82,18 \pm 3,57$ & $<0,001$ \\
\hline Envergadura (cm) & $164,52 \pm 6,85$ & $161,86 \pm 5,13$ & 0,130 \\
\hline D Biacromial (cm) & $35,27 \pm 1,70$ & $35,59 \pm 1,57$ & 0,554 \\
\hline D Biileocrestal (cm) & $28,95 \pm 3,57$ & $27,17 \pm 1,56$ & 0,076 \\
\hline D Trasnverso del tórax (cm) & $26,97 \pm 1,56$ & $26,05 \pm 2,08$ & 0,116 \\
\hline D Anteroposterior del tórax & $17,87 \pm 1,62$ & $17,09 \pm 1,23$ & 0,113 \\
\hline D Biepicondíleo del húmero & $6,17 \pm 0,34$ & $6,18 \pm 0,23$ & 0,868 \\
\hline D Bicondíleo del fémur (cm) & $9,00 \pm 0,58$ & $8,98 \pm 0,42$ & 0,865 \\
\hline D Biestiloideo $(\mathrm{cm})$ & $5,06 \pm 0,33$ & $4,96 \pm 0,25$ & 0,254 \\
\hline PR Brazo relajado (cm) & $25,88 \pm 2,38$ & $25,08 \pm 2,99$ & 0,300 \\
\hline PR Brazo corregido (cm) & $21,15 \pm 1,35$ & $20,63 \pm 2,47$ & 0,357 \\
\hline PR Brazo contraído (cm) & $27,68 \pm 2,15$ & $26,55 \pm 2,06$ & 0,063 \\
\hline PR Mesoesternal (cm) & $85,83 \pm 4,97$ & $82,48 \pm 5,39$ & $\mathbf{0 , 0 2 7}$ \\
\hline PR Cintura $(\mathrm{cm})$ & $68,92 \pm 5,79$ & $65,85 \pm 5,64$ & 0,064 \\
\hline PR Cadera $(\mathrm{cm})$ & $91,14 \pm 7,44$ & $89,69 \pm 5,92$ & 0,450 \\
\hline PR Muslo medio (cm) & $47,98 \pm 4,30$ & $46,11 \pm 4,46$ & 0,138 \\
\hline PR Muslo corregido (cm) & $41,11 \pm 3,67$ & $39,41 \pm 3,81$ & 0,018 \\
\hline PR Pierna (máx.) (cm) & $33,61 \pm 2,38$ & $32,11 \pm 2,25$ & $\mathbf{0 , 0 2 7}$ \\
\hline PR Pierna corregido $(\mathrm{cm})$ & $28,57 \pm 2,33$ & $27,07 \pm 1,97$ & 0,116 \\
\hline
\end{tabular}

Datos expresados como media \pm desviación típica. PR: perímetro. D: diámetro. 
Tabla II. Comparación y descripción de la proporcionalidad (Valores Z) de las mujeres kayakistas jóvenes de aguas tranquilas y slalom.

\begin{tabular}{|c|c|c|c|}
\hline Variable & $\begin{array}{l}\text { Aguas Tranquilas } \\
\qquad(n=26)\end{array}$ & $\begin{array}{c}\text { Slalom } \\
(n=24)\end{array}$ & Valor $-p$ \\
\hline Z Peso & $-0,19 \pm 0,63$ & $0,20 \pm 1,10$ & 0,132 \\
\hline$Z$ Sumatorio de 6 pliegues & $-0,46 \pm 0,67$ & $-0,54 \pm 0,83$ & 0,716 \\
\hline$Z$ Sumatorio de 8 pliegues & $-0,47 \pm 0,69$ & $-0,58 \pm 0,82$ & 0,619 \\
\hline$Z$ Talla sentado & $0,01 \pm 0,43$ & $-0,31 \pm 0,71$ & 0,060 \\
\hline$Z$ Envergadura & $-0,21 \pm 0,39$ & $0,26 \pm 0,49$ & $<0,001$ \\
\hline Z D Biacromial & $-0,73 \pm 0,81$ & $0,06 \pm 0,73$ & $\mathbf{0 , 0 0 3}$ \\
\hline Z D Biile ocrestal & $0,67 \pm 1,84$ & $0,17 \pm 0,93$ & 0,327 \\
\hline Z D Trasnverso del tórax & $0,04 \pm 0,58$ & $0,00 \pm 1,21$ & 0,891 \\
\hline Z D Anteroposterior del tórax & $0,76 \pm 1,04$ & $0,59 \pm 0,95$ & 0,624 \\
\hline Z D Biepicondíleo del húmero & $-0,22 \pm 0,98$ & $0,50 \pm 0,73$ & 0,005 \\
\hline Z D Bicondíleo del fémur & $-0,36 \pm 1,00$ & $0,32 \pm 0,92$ & 0,016 \\
\hline Z D Biestiloideo & $0,14 \pm 1,02$ & $0,49 \pm 0,95$ & 0,225 \\
\hline Z PR Brazo relajado & $-0,02 \pm 0,80$ & $0,06 \pm 1,45$ & 0,796 \\
\hline Z PR Brazo corregido & $-0,05 \pm 0,54$ & $0,10 \pm 1,46$ & 0,626 \\
\hline Z PR Brazo contraído & $-0,29 \pm 0,70$ & $-0,34 \pm 1,00$ & 0,846 \\
\hline Z PR Mesoesternal & $0,24 \pm 0,66$ & $0,19 \pm 1,15$ & 0,876 \\
\hline$Z$ PR Cintura & $-0,10 \pm 1,01$ & $-0,22 \pm 1,39$ & 0,726 \\
\hline Z PR Cadera & $-0,03 \pm 0,98$ & $0,35 \pm 1,14$ & 0,216 \\
\hline Z PR Muslo medio & $-0,75 \pm 0,80$ & $-0,39 \pm 2,12$ & 0,932 \\
\hline Z PR Muslo corregido & $0,68 \pm 1,01$ & $0,65 \pm 1,34$ & 0,937 \\
\hline Z PR Pierna (máx.) & $-0,16 \pm 0,81$ & $-0,28 \pm 1,18$ & 0,694 \\
\hline Z PR Piern a corregido & $-0,29 \pm 1,04$ & $-0,53 \pm 1,18$ & 0,451 \\
\hline
\end{tabular}

Datos expresados como media \pm desviación típica. PR: perímetro. D: diámetro.

ferior de aproximadamente $4 \mathrm{~kg}$, así como una menor adiposidad que las de aguas tranquilas, con diferencias de $10 \mathrm{y}$ $6 \mathrm{~mm}$ en los sumatorios de 8 y 6 pliegues, respectivamente. Resultado indicado en el mismo sentido por Ridge et al., al comparar estas mismas especialidades en mujeres kayakistas que participaron en los Juegos Olímpicos de Sydney.

A pesar de las diferencias encontradas en la talla, los valores del índice de masa corporal fueron similares en ambos grupos de palistas $(21,22 \pm 2,92$ y $20,95 \pm 2,15 \mathrm{~kg}$. $\mathrm{m}-2$, para las kayakistas de slalom y aguas tranquilas, respectivamente). Sin embargo, las palistas de aguas tranquilas obtuvieron valores significativamente superiores a las de slalom en los perímetros mesoesternal, así como del muslo corregido y de la pierna, observándose cierta tendencia en el mismo sentido en los perímetros del brazo contraído $(\mathrm{p}<0,063)$ y la cintura $(\mathrm{p}<0,064)$. Estos resultados, en lo referente a los perímetros mesoesternal y del brazo contraído, coinciden con las características frecuentemente descritas de los palistas de aguas tranquilas de elite (Ackland et al., 2003; Aitken \& Jenkins, 1998; Fry \& Morton, 1991; MisigojDurakovic \& Heimer; Shephard, 1987; van Someren \& Palmer, 2003); mientras que las diferencias en los perímetros del miembro inferior podrían estar relacionadas con una mayor actividad de las piernas en aguas tranquilas al realizar un movimiento más dinámico de pedaleo, aunque no parece de mayor intensidad que el trabajo de carácter isométrico de control de la embarcación desarrollado con las extremidades inferiores en slalom.

Las características en cuanto a proporcionalidad de las mujeres kayakistas estudiadas fueron similares (Tabla 
Tabla III. Comparación y descripción del somatotipo, porcentaje de grasa, masa libre de grasa y años desde/hasta la edad de máxima velocidad de crecimiento.

\begin{tabular}{lccc}
\hline Variable & Aguas Tranquilas (n=26) & Slalom $(\mathbf{n = 2 4})$ & Valor $\boldsymbol{- p}$ \\
\hline Endomorfia & $4,0 \pm 1,1$ & $3,8 \pm 1,1$ & 0,527 \\
Mesomorfia & $3,8 \pm 0,7$ & $4,1 \pm 1,0$ & 0,196 \\
Ectomorfia & $2,8 \pm 0,9$ & $2,4 \pm 1,4$ & 0,247 \\
& & & 0,191 \\
Porcentaje de grasa corporal & $22,45 \pm 5,83$ & $20,39 \pm 5,13$ & 0,198 \\
Masa libre de grasa (kg) & $43,51 \pm 4,54$ & $41,89 \pm 4,18$ & $\mathbf{0 , 0 1 0}$ \\
& & & $1,35 \pm 0,45$ \\
Años hasta/desde la edad de máxima & $1,73 \pm 0,54$ & &
\end{tabular}

Datos expresados como media \pm desviación típica.

II), excepto en los valores $\mathrm{Z}$ de la envergadura y diámetros biacromial, biepicondíleo del húmero y bicondíleo del fémur, con valores significativamente superiores para las palistas de slalom. Estas diferencias pueden ser atribuidas a que todas estas medidas de proporcionalidad son obtenidas tomando como referencia las proporciones de un modelo de referencia (Phantom) y la talla del sujeto a estudiar. Por ello, cuando los valores absolutos de una variable son similares

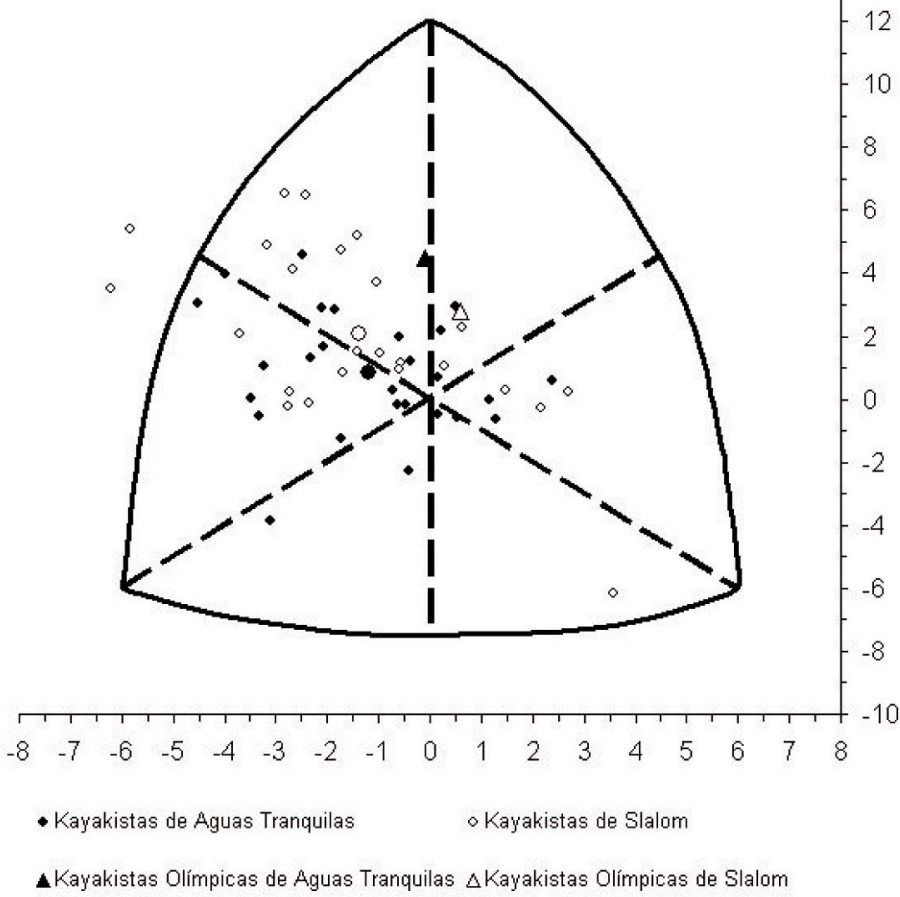

Fig. 1. Somatopuntos de las mujeres kayakistas de aguas tranquilas y slalom y su comparación con kayakistas de categoría olímpicas (Ackland et al., 2003; Ridge et al.). Los círculos grandes representan el valor medio. en ambos grupos, el valor $\mathrm{Z}$ adquiere una puntuación superior en la población de menor talla. Similares valores de proporcionalidad también han sido encontrados en palistas hombres de aguas tranquilas y slalom de categoría olímpica (Ridge et al.).

El somatotipo medio de ambos grupos se corresponde con el morfotipo mesomorfo endomorfo, siendo muy similares ya que no se encontraron diferencias significativas al analizar cada uno de los componentes de forma independiente (Tabla III), ni al calcular la distancia de dispersión de los somatotipos medios, arrojando un valor de 1,29. En la figura 1 se representan cada uno de los somatopuntos, el somatotipo medio y el correspondiente a mujeres kayakistas de elite. Se puede observar una mayor heterogeneidad en el grupo de palistas de slalom, lo que se confirma con un resultado de 1,72 puntos en la dispersión morfogénica media del somatotipo (somatotype attitudinal mean), entendida como una medida de la dispersión media de los somatotipos individuales respecto al somatotipo medio, frente a los 1,44 obtenidos por las kayakistas de aguas tranquilas. En ambos casos, los resultados quedan lejos de los 1,0 puntos obtenidos por mujeres kayakistas olímpicas de aguas tranquilas (Ackland et al., 2003), aunque son cercanos a los 1,27 y 1,66 descritos en mujeres kayakistas de 13 y 14 años, respectivamente (Alacid et al., 2011b)

Los porcentajes de grasa oscilaron del 20 al $22 \%$ en cada uno de los grupos, valores similares a los descritos en mujeres kayakistas de aguas tranquilas de elite (Bishop, 2000), de categoría juvenil (Gobbo et al., 2002), infantil (Cuesta et al., 1991) y de elite en slalom (Sidney \& Shephard, 1973). De cualquier forma, todas estas comparaciones deben interpretarse con cautela debido a la gran variedad de fórmulas, plie- 
gues seleccionados y técnicas utilizadas en la obtención de los mismos para el cálculo posterior del porcentaje de grasa.

Como conclusión, este trabajo aporta una importante cantidad de información normativa sobre las características antropométricas de las mujeres kayakistas de aguas tranquilas y slalom que pueden utilizarse de referencia en el proceso de valoración y entrenamiento. Las palistas de aguas tranquilas se caracterizaron por una mayor talla y talla sentado que las de slalom, diferencias que pueden ser interpretadas como un mayor desarrollo madurativo de las primeras. Los valores de proporcionalidad fueron similares en la mayoría de las variables analizadas, pudiéndose justificar la mayor envergadura y diámetros biacromial, biepicondíleo del húmero y bicondíleo del fémur en las palistas de slalom por las diferencias en el valor absoluto de la talla anteriormente descrito. Ambos grupos de kayakistas presentaron un somatotipo medio muy similar, clasificado como mesomorfo endomorfo, siendo las palistas de slalom las que presentaron una mayor heterogeneidad en cuanto a la dispersión del somatotipo.

\section{AGRADECIMIENTOS}

Los autores agradecen su colaboración a la Real Federación Española de Piragüismo, así como a los entrenadores y deportistas que participaron en el estudio.

ALACID, F.; MUYOR, J. M.; VAQUERO, R. \& LÓPEZ-MIÑARRO, P. A. Morphological characteristics and maturity status of young female sprint and slalom kayakers. Int. J. Morphol., 30(3):895-901, 2012.

SUMMARY: The aim of this study was to describe and compare the kinanthropometric characteristics, maturity status, somatotype and proportionality between young female sprint and slalom kayakers. Fifty young female kayakers (26 sprint; 24 slalom), aged 13 and 14 years old, were assessed using a battery of 29 anthropometric dimensions. Body fat, somatotypes, Phantom Z-scores and maturity status expressed as the time to/from the age of peak height velocity were calculated. Sprint kayakers were taller and had a higher sitting height than slalom kayakers, these differences could be explained by the greater maturity status in the sprint paddlers group. Proportionality values were similar in most of the variables analysed. Mean somatotypes were best described as mesomorphy endomorph in both groups, while slalom kayakers exhibited a higher heterogeneity. The data provided in this study could be used as a guideline for talent identification in sprint and slalom female kayakers.

\section{KEY WORDS: Anthropometry, Somatotype; Proportionality; Canoeing.}

\section{REFERENCIAS BIBLIOGRÁFICAS}

Ackland, T. R.; Ong, K. B.; Kerr, D. A. \& Ridge, B. Morphological characteristics of Olympic sprint canoe and kayak paddlers. $J$. Sci. Med. Sport, 6(3):285-94, 2003.

Ackland, T. R.; Schreiner, A. B. \& Kerr, D. A. Absolute size and proportionality characteristics of World Championship female basketball players. J. Sports Sci., 15(5):485-90, 1997.

Aitken, D. A. \& Jenkins, D. G. Anthropometric-based selection and sprint kayak training in children. J. Sports Sci., 16(6):53943, 1998.

Alacid, F.; López-Miñarro, P. A.; Martínez, I. \& Ferrer-López, V. Anthropometric indexes in young paddlers. Rev. Int. Med. Cienc. Ac., 10(41):58-76, $2011 \mathrm{a}$.

Alacid, F.; Marfell-Jones, M.; López-Miñarro, P. A.; Martínez, I. \& Muyor, J. M. Morphological characteristics of young elite paddlers. J. Hum. Kinet., 27:95-110, 2011b.

Alacid, F.; Muyor, J. M. \& López-Miñarro, P. A. Perfil antropométrico del canoísta joven de aguas tranquilas. Int. J. Morphol., 29(3):835-40, 2011.
Bishop, D. Physiological predictors of flat-water kayak performance in women. Eur. J. Appl. Physiol., 82(1-2):91-7, 2000.

Carrascosa, A.; Fernández, J. M.; Fernández, C.; Ferrández, A.; López-Siguero, J. P.; Sánchez, E.; Sobradillo, B. \& Yeste, D. Spanish cross-sectional growth study 2008. Part II. Height, weight and body mass index values from birth to adulthood. An. Pediatr. (Barc), 68(6):552-69, 2008.

Carter, J. E.; Ackland, T. R.; Kerr, D. A. \& Stapff, A. B. Somatotype and size of elite female basketball players. J. Sports Sci., 23(10):1057-1063, 2005.

Carter, J. E. L. \& Ackland, T. Kinanthropometry in aquatic sports. A study of world class athletes. Champaign, Human Kinetics, 1994.

Carter, J. E. L. \& Heath, B. H. Somatotyping: development and application. Cambridge, Cambridge University Press, 1990.

Cuesta, G.; Polo, J. M. \& Padilla, S. Correlación entre la marca deportiva obtenida en test de campo y parámetros fisiológicos obtenidos en laboratorio, en piragüistas adolescentes. Apunts, 18:130-42. 1991. 
Duncan, M. J.; Woodfield, L. \& al-Nakeeb, Y. Anthropometric and physiological characteristics of junior elite volleyball players. Br. J. Sports Med., 40(7):649-51, 2006.

Fry, R. W. \& Morton, A. R. Physiological and kinanthropometric attributes of elite flatwater kayakists. Med. Sci. Sports Exerc., 23(11):1297-301, 1991.

Gobbo, L. A.; Papst, R. R.; Carvalho, F. O.; Souza, C. F.; Cuattrin, S. A. \& Cyrino, E. S. Perfil antropométrico da seleção brasileira de canoagem. Rev. Bras. Cs. e Mov., 10(1):7-12, 2002.

Goncetas, A. \& Landor, A. Morphological and physiological parameters in relation to playing position of high level basketball players. Paper Anthropol., 14:42-52, 2005.

Hencken, C. \& White, C. Anthropometric assessment of Premiership soccer players in relation to play position. Eur. $J$. Sport Sci., 6(4):205-11, 2006.

Hirata, K. Selections of olympic champions. Tokio, Hirata Institute, 1977.

Jürimäe, J. \& Jürimäe, T. Differences in anthropometric and physical performance characteristics between lightweight and open class rowers. Paper Anthropol., 11:71-80. 2002.

Kerr, D. A.; Ross, W. D.; Norton, K.; Hume, P.; Kagawa, M. \& Ackland, T. R. Olympic lightweight and open-class rowers possess distinctive physical and proportionality characteristics. J. Sports Sci., 25(1):43-53, 2007.

Marfell-Jones, M.; Olds, T.; Stewart, A. \& Carter, L. International standards for anthropometric assessment. Potchefstroom, South Africa, ISAK, 2006.

Mirwald, R. L.; Baxter-Jones, A. D.; Bailey, D. A. \& Beunen, G. P. An assessment of maturity from anthropometric measurements. Med. Sci. Sports Exerc., 34(4):689-94, 2002.

Misigoj-Durakovic, M. \& Heimer, S. Characteristics of the morphological and functional status of kayakers and canoeists. J. Sports Med. Phys. Fitness, 32(1):45-50, 1992.

RFEP. (2010). Reglamento General y Técnico de Competiciones. Descargado el 20-12-2010, 2010, desde www.rfep.es.

Ridge, B.; Broad, E.; Kerr, D. \& Ackland, T. Morphological characteristics of Olympic slalom canoe and kayak paddlers. Eur. J. Sport Sci., 7(2):107-13, 2007.

Rodriguez, G.; Moreno, L. A.; Blay, M. G.; Blay, V. A.; Fleta, J.; Sarria, A. \& Bueno, M. Body fat measurement in adolescents: comparison of skinfold thickness equations with dual-energy X-ray absorptiometry. Eur. J. Clin. Nutr., 59(10):1158-66, 2005.

Ross, W. D. \& Marfell-Jones, M. Kinanthropometry. En J. MacDougal, H. Wenger \& H. Green (Eds.), Physiological testing of the high performance athlete. 2 ed., pp. 223-308, Champaign (IL), Human Kinetics, 1991.

Shephard, R. J. Science and medicine of canoeing and kayaking. Sports Med., 4(1):19-33, 1987.

Sidney, K. \& Shephard, R. J. Physiological characteristics and performance of white-water paddler. Eur. J. Appl. Physiol. Occup. Physiol., 32(1):55-70, 1973.

Slaughter, M. H.; Lohman, T. G.; Boileau, R. A.; Horswill, C. A.; Stillman, R. J.; Van Loan, M. D. \& Bemben, D. A. Skinfold equations for estimation of body fatness in children and youth. Hum. Biol., 60(5):709-23, 1988.

Sutton, L.; Scott, M.; Wallace, J. \& Reilly, T. Body composition of English Premier League soccer players: influence of playing position, international status, and ethnicity. J. Sports Sci., 27(10):1019-26, 2009.

Toledo, C. L.; Roquetti, P. \& Fernandes, J. Análisis del perfil antropométrico de jugadores de la selección brasileña de voleibol infanto juvenil. Int. J. Morphol., 28(4):1035-41, 2010.

van Someren, K. A. \& Palmer, G. S. Prediction of 200-m sprint kayaking performance. Can. J. Appl. Physiol., 28(4):505-17, 2003.

Vucetic, V.; Matkovic, B. R. \& Sentija, D. Morphological differences of elite Croatian track-and-field athletes. Coll. Antropol., 32(3):863-8, 2008.

\section{Dirección para correspondencia: \\ Fernando Alacid Cárceles}

Facultad de Ciencias de la Actividad Física y el Deporte

Universidad Católica San Antonio

Campus de los Jerónimos s/n.

30107 - Guadalupe

Murcia

ESPAÑA.

Tel. (+34) 968278824

Email: falacid@ucam.edu

Received: 11-07-2011

Accepted: 14-05-2012 